\title{
Estudo da transmissão da leishmaniose tegumentar americana no Distrito Federal
}

\author{
Study on the transmission of American cutaneous \\ leishmaniasis in the Federal District
}

\author{
Raimunda Nonata Ribeiro Sampaio ${ }^{1}$, Marianne de Castro Gonçalves ${ }^{1}$, \\ Viviane Alves Leite ${ }^{1}$, Bruna Valle França ${ }^{1}$, Gilcilene Santos ${ }^{1}$, \\ Maria do Socorro Laurentino Carvalho ${ }^{2}$ e Pedro Luiz Tauil ${ }^{3}$
}

\begin{abstract}
RESUMO
O objetivo do estudo foi caracterizar a leishmaniose tegumentar em pacientes do Distrito Federal, investigar infecção subclínica nos moradores das localidades dos pacientes e identificar as espécies de flebotomíneos e leishmanias. Foram selecionados pacientes atendidos no Hospital Universitário de Brasília de agosto de 2006 a junho de 2007. Parentes e vizinhos dos mesmos foram submetidos à intradermorreação de Montenegro e imunofluorescência indireta. Foram capturados flebotomíneos nas localidades de origem dos pacientes e identificados quanto às espécies, bem como foram identificadas as espécies de leishmanias encontradas nos pacientes. Foram registrados 10 casos autóctones de leishmaniose tegumentar. Em 32 moradores, foi realizada intradermorreação, com positividade de 71,8\%. Trinta e sete imunofluorescências realizadas foram negativas. Foram capturadas Lutzomyia whitmani, inclusive no domicílio/peridomicílio e Lutzomyia flaviscutellata. 0 percentual de positividade das intradermorreações de Montenegro sugere infecção subclínica dos moradores. A captura do vetor Lutzomyia whitmani no peri/intradomicílio sugere transmissão peri/intradomicíliar.
\end{abstract}

Palavras-chaves: Epidemiologia. Leishmaniose cutânea. Leishmania (Viannia) braziliensis. Lutzomyia whitmani. Distrito Federal.

\begin{abstract}
The objectives of this study were to characterize cutaneous leishmaniasis in patients from the Federal District, investigate subclinical infection in people living in the same localities as patients and identify the species of Phlebotomus and Leishmania. Patients attended at the University Hospital of Brasília between August 2006 and June 2007 were selected. Relatives and neighbors of the patients underwent the Montenegro intradermal test and indirect immunofluorescence. Phlebotomines were caught at the localities where the patients came from and their species were identified. The species of Leishmania in the patients were also identified. Ten autochthonous cases of cutaneous leishmaniasis were recorded. The intradermal test was done on 32 local residents and $71.8 \%$ were positive. Immunofluorescence was performed on 37 individuals and all of them were negative. Lutzomyia whitmani was caught, including in domestic/peridomestic areas, along with Lutzomyia flaviscutellata. The percentage of positive Montenegro intradermal tests suggests that the local residents had subclinical infection. Capture of the vector Lutzomyia whitmani in domestic/peridomestic areas suggests that domestic/peridomestic transmission was occurring.
\end{abstract}

Key-words: Epidemiology. Cutaneous leishmaniasis. Leishmania (Viannia) braziliensis. Lutzomyia whitmani. Federal District.

O Brasil é um dos cinco países com maior número de casos de leishmaniose tegumentar nas Américas, destacando-se nele as regiões Norte, Nordeste e Centro-Oeste. Entre 1985 e 2005, o Ministério da Saúde notificou média anual de 28.568 novos casos de LTA com taxas de incidência nos estados variando entre 3,8 a 22,9 por 100.000 habitantes. A região Centro-Oeste figurou como a terceira em incidência e a primeira em crescimento de LTA ${ }^{10}$.

1. Laboratório de Dermatomicologia, Faculdade de Medicina, Universidade de Brasilia. Brasília, DF. 2. Diretoria de Vigilância Ambiental em Saúde, Secretaria do Estado de Saúde do Distrito Federal. Brasília, DF. 3. Área de Medicina Social, Faculdade de Medicina, Universidade de Brasília. Brasília, DF.

Apoio financeiro: FINATEC

Endereço para correspondência: Prof ${ }^{a}$ Raimunda Nonata Ribeiro Sampaio. SHIS QI 25, conj. 2, casa 1, 71660-220 Brasília, DF.

Tel: $55613367-1331$

e-mail: rsampaio@unb.br

Recebido para publicação em 30/12/2008

Aceito em 08/10/2009
No Distrito Federal (DF) há evidência de transmissão autóctone de $\mathrm{LTA}^{12}{ }^{15}{ }^{13}$. Sampaio e cols ${ }^{15}$ relataram em 19800 primeiro caso de origem do DF em criança de 2 anos do Núcleo Bandeirante que nunca havia saído do DF. Em 1999, foram notificados 11 casos novos ${ }^{1314}$. Em 2004 a Universidade de Brasília estudou um surto na Cidade de São Sebastião, DF. De acordo com os dados do Ministério da Saúde ${ }^{9}$ o número de casos notificados, procedentes do DF, nos anos de 2003, 2004 e 2005 foi de 31, 57 e 26, respectivamente.

A intradermorreação de Montenegro (IDRM) detecta a presença de hipersensibilidade tardia aos antígenos de leishmania e tem grande valor presuntivo no diagnóstico de LTA. Desta forma, é muito útil no levantamento de inquéritos epidemiológicos de áreas endêmicas ${ }^{1718}$.

A reação de imunofluorescência indireta (IFI) é uma técnica sensível para o diagnóstico de LTA, é habitualmente positiva nas 
formas cutânea difusa, mucosa, disseminada e pode ser positiva em pacientes com lesões cutâneas recentes. Tem também grande utilidade em levantamento de infecção subclínica por leishmania ${ }^{717}$.

Este estudo teve como finalidade aprofundar o estudo da autoctonia LTA no DF caracterizando os casos clínicos autóctones, investigando infecções subclínicas nas localidades onde viviam os pacientes e capturando e identificando as espécies de flebotomíneos e leishmanias isoladas.

\section{PACIENTES E MÉTODOS}

Tipo de estudo. Trata-se de um estudo clínico, laboratorial e epidemiológico, descritivo, prospectivo do tipo série de casos.

Geografia da região. 0 Distrito Federal está localizado entre paralelos $15^{\circ} 30^{\prime}$ e $16^{\circ} 03^{\prime}$ de latitude sul, entre o Rio Preto, a leste, e o Rio Descoberto, a oeste. Sua área é de $5.789 \mathrm{~km}$, altitude média de $1.100 \mathrm{~m}$, com o relevo caracterizado pela presença de todos os tipos de vegetação normalmente englobados no cerrado. 0 clima da região é do tipo tropical de savana e temperado chuvoso. A temperatura média e de $20,5^{\circ} \mathrm{C}\left(69^{\circ} \mathrm{F}\right)$. A umidade relativa do ar é baixa, variando entre $25 \%$ durante o inverno e $68 \%$ no verão.

O Distrito Federal é dividido em 29 regiões administrativas (RA) oficiais: Plano Piloto, Gama, Taguatinga, Brazlândia, Sobradinho, Planaltina, Paranoá, Núcleo Bandeirante, Ceilândia, Guará, Cruzeiro, Samambaia, Santa Maria, Agrovila São Sebastião, Recanto das Emas, Lago Sul, Riacho Fundo, Lago Norte, Candangolândia, Águas Claras, Riacho Fundo II, Varjão, Sudoeste/Octogonal, Park Way, Setor Complementar de Indústria e Abastecimento (Estrutural), Sobradinho II, Setor de Indústria e Abastecimento (SAI), Jardim Botânico e Itapo $\tilde{a}^{20}$.

Casos humanos. De agosto de 2006 a junho de 2007, foram selecionados casos de LTA que procuraram o Hospital Universitário de Brasília (HUB), observando-se rigorosamente, o pré-requisito de serem incluídos apenas os que informaram, por meio de sua história, não haverem saído dos limites do DF por um tempo mínimo de seis meses precedentes ao início da lesão cutânea.

0 estudo seguiu as diretrizes estabelecidas pela Resolução do Conselho Nacional de Saúde n 196/96 (declaração de Helsinki) sobre normas de pesquisa envolvendo seres humanos.

0 diagnóstico de leishmaniose foi feito pela história, exame dermatológico e pela positividade de, pelo menos, dois exames diagnósticos específicos ${ }^{3}$.

Para realizar a IDRM, inoculou-se $0,1 \mathrm{ml}$ do antígeno padronizado em $40 \mathrm{ugN} / \mathrm{ml}$ por via intradérmica na face anterior do antebraço esquerdo e realizou-se a leitura após 48h, considerando-se positiva quando houve pápula maior ou igual a $5 \mathrm{~mm}^{10}$.

Para a realização da IFI foram colhidos $4 \mathrm{ml}$ de sangue, posteriormente centrifugados, e mantido o soro em freezer a $-80^{\circ} \mathrm{C}$ até 0 momento do exame. Considerou-se positiva a reação a partir da diluição 1:40.

Além disto, foram realizados: pesquisa de amastigotas em esfregaço, cultura para leishmania, exame histopatológico das lesões e identificação de leishmania por reação em cadeia de polimerase e anticorpos monoclonais ${ }^{356}$.

Estudo dos contatos nos locais prováveis de infecção. Uma equipe de pesquisadores e técnicos da SES, DF visitou os locais de provável aquisição da doença pelos pacientes. Nestes locais, foram realizadas IDRM e IFI em todos os parentes e moradores residentes num raio de até $1 \mathrm{~km}$ do local de moradia do caso-índice.

Os vizinhos moradores, voluntários, foram previamente esclarecidos e orientados, quanto ao tipo de exame a ser realizado e quanto ao seu objetivo e participaram, livremente, após assinatura de termo de consentimento esclarecido, pós-informado, dentro das disposições éticas já citadas. Foram submetidos à coleta de $4 \mathrm{ml}$ de sangue para IFI e à inoculação do antígeno de Montenegro, com leitura após 48 horas, procedimentos semelhantes aos realizados nos pacientes.

Estudo entomológico. No período de maio de 2006 a junho de 2007, uma equipe composta por pesquisadores e técnicos da Diretoria de Vigilância Ambiental da Secretaria de Saúde do Governo do Distrito Federal (SES, DF) foi enviada à área selecionada para realizar a captura dos flebotomíneos. Esta foi realizada com armadilha luminosa tipo CDC por 3 noites consecutivas, em diferentes tipos de ambientes, em áreas rurais das regiões administrativas de São Sebastião, Gama e Planaltina (regiões de origem dos pacientes), no intra e peridomicílio (abrigo de animais) e em diferentes fitofisionomias de cerrado.

Os exemplares capturados foram transportados vivos ao laboratório. Neste, foram armazenados em congelador para imobilização e posterior separação dos flebotomíneos dos demais insetos. Após fixação adequada, foi realizada identificação de sexo e espécie.

Comitê de ética. 0 estudo foi aprovado pelo Comitê de Ética em Pesquisa da Faculdade de Medicina da Universidade de Brasília (parecer número 046/2006).

\section{RESULTADOS}

Entre agosto de 2006 e junho de 2007, foram atendidos no HUB 10 casos de LTA procedentes do DF. Considerando as regiões administrativas, quatro pacientes procediam de Planaltina, três do Gama, um de São Sebastião, um do Paranoá e um da Ceilândia (Figura 1).

A razão de gênero dos casos foi de 9:1, com predomínio do sexo masculino. A idade dos pacientes variou de 11 a 58 anos, com média de 34,5 $\pm 15,37$ e mediana de 37 anos.

A Tabela 1 informa aspectos clínicos e os exames laboratoriais dos 10 pacientes com LTA adquirida no Distrito Federal. Todos os pacientes com leishmaniose cutânea apresentaram lesão ulcerada única localizada preferencialmente nos membros superiores (seis), seguidos dos membros inferiores (dois), cabeça (um) e abdome (um). Dos 10 pacientes do estudo, seis deles chegaram ao hospital com até três meses de duração da lesão (Tabela 1). 
TABELA 1

Aspectos clínicos e exames laboratoriais dos 10 pacientes com leishmaniose tegumentar americana adquirida no Distrito Federal, no período de agosto de 2006 a junho de 2007.

\begin{tabular}{|c|c|c|c|c|c|c|c|c|c|c|}
\hline REG & Sexo & $\begin{array}{l}\text { Idade } \\
\text { (anos) }\end{array}$ & Local de infecção & Local & Tempo (meses) & IDRM & IFI & Cultura & amastigotas & $\begin{array}{c}\text { Espécie de } \\
\text { Leishmania Viannia }\end{array}$ \\
\hline 286419 & M & 33 & Planaltina & MMSS* & 4 & + & + & + & + & braziliensis \\
\hline 66874 & M & 12 & Planaltina & Cabeça & 3 & + & + & + & - & braziliensis \\
\hline 70378 & M & 11 & São Sebastião & MMII* & 4 & + & + & + & - & não identificada \\
\hline 71001 & M & 58 & Planaltina & MMII & 3 & + & - & + & + & braziliensis \\
\hline 69820 & M & 35 & Gama & MMSS & 9 & + & + & - & + & braziliensis \\
\hline 76625 & M & 28 & Paranoá & MMSS & 1 & + & + & - & - & não identificada \\
\hline 76631 & $\mathrm{~F}$ & 44 & Gama & abdome & 1 & + & - & + & + & não identificada \\
\hline 76628 & M & 42 & Gama & MMSS & 6 & + & - & + & + & braziliensis \\
\hline 273189 & M & 39 & Ceilândia & MMSS & 3 & + & + & + & - & não identificada \\
\hline 68690 & M & 43 & Planaltina & MMSS & 2 & + & - & - & + & não identificada \\
\hline
\end{tabular}

*MMSS: membros superiores, MMII: membros inferiores, REG: registro hospitalar, IDRM: intradermorreação de Montenegro, IFI: imunofluorescência indireta.

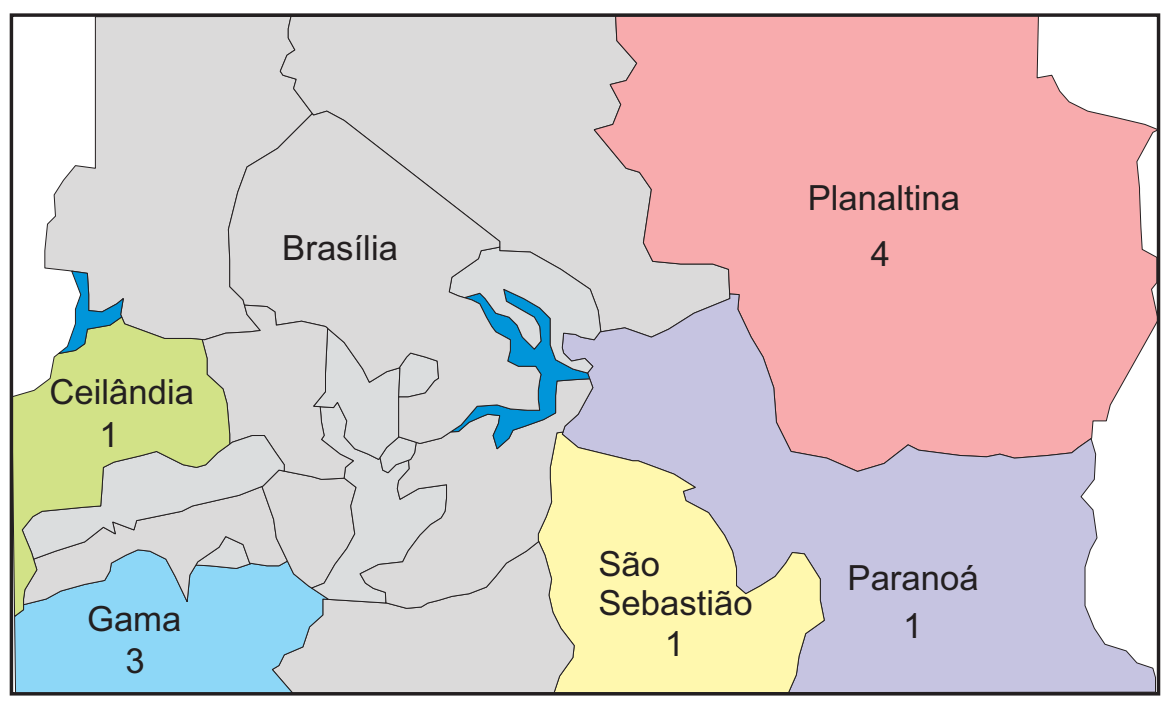

FIGURA 1

Distribuição geográfica de 10 casos de leishmaniose cutânea atendidos no Hospital Universitário de Brasília, procedentes de cinco regiões administrativas do Distrito Federal entre agosto de 2006 a junho de 2007.

Todos pacientes tiveram IDRM positiva e seis tiveram IFI positivas. A pesquisa de amastigotas em esfregaço foi positiva em seis pacientes. A cultura foi positiva em sete e este resultado coincidiu com a pesquisa de amastigotas em quatro casos. A presença de leishmania no exame histopatológico foi negativa em todos os casos. Nove, de 10 biópsias, apresentaram infiltrado linfohistio-plasmocitário compatível com LTA. Cinco dos 10 pacientes tiveram culturas identificadas como Leishmania (Viannia) braziliensis (Tabela 1).

Dos 10 pacientes, oito foram tratados com N-metil glucamina (NMG) endovenoso, 20mgSbV/kg/dia, durante 20 dias. Dois, que apresentaram alteração eletrocardiográfica prévia, foram tratados com NMG intralesional. Todos tiveram as lesões cicatrizadas.

Foram visitados parentes, moradores da mesma casa e vizinhos de quatro pacientes. Duas residências eram localizadas no Gama, uma em São Sebastião e outra em Planaltina.

Trinta e dois moradores foram submetidos à intradermorreação de Montenegro, sendo 59,3\% provenientes da RA Gama, 21,8\% da RA São Sebastião e 18,7\% da RA Planaltina. Destes, 71,8\% apresentaram IDRM positiva ( $\geq 5 \mathrm{~mm}$ ), sendo $60,8 \%$ indivíduos do sexo feminino e 39,2\% do sexo masculino, com idade entre 7 e 45 anos, média de 21,16 $\pm 14,01$ e mediana de 19 anos. Detectou-se 21,7\% das IDRM positivas pertenciam a crianças com idade menor ou igual 10 anos. 0 percentual de positividade da IDRM foi de $71,4 \%$ na RA São Sebastião, 68,4\% na RA Gama e 83,3\% na RA Planaltina (Figura 2).

Foram realizadas IFI em 37 contatos, sendo 64,8\% provenientes da RA Gama, 16,2\% da RA Planaltina e 21,8\% da RA São Sebastião. Todas IFI foram negativas.

Quanto às capturas de flebotomíneos realizadas durante o período de maio 2006 a junho 2007, as duas espécies encontradas foram Lutzomyia whitmani e Lutzomyia flaviscutellata. Lutzomyia whitmani foi a espécie encontrada com maior frequência no DF, distribuindo-se nas regiões administrativas São Sebastião (60,4\%), Gama $(27,8 \%)$ e Planaltina (11,8\%) (Tabela 2). Quanto ao local de coleta, 59,2\% foram coletadas no peridomicílio, 4,1\% no intradomicilio e 36,7\% no cerrado. A Lutzomyia flaviscutellata só foi encontrada na vegetação (Tabela 3). 
TABELA 2

Distribuição das espécies de flebotomíneos vetores da leishmaniose tegumentar, no Distrito Federal, no período de maio de 2006 a junho de 2007.

\begin{tabular}{lrrrrrr}
\hline & \multicolumn{2}{c}{ Lutzomyia } & & \multicolumn{2}{c}{ Lutzomyia } \\
Regiões & \multicolumn{2}{c}{ whitmani } & & \multicolumn{2}{c}{ flaviscutellata } & Total \\
\cline { 2 - 3 } Administrativas & $\mathrm{n}^{0}$ & $\%$ & & $\mathrm{n}^{0}$ & $\%$ & $\mathrm{n}^{0}$ \\
\hline São Sebastião & 154 & 60,4 & & 0 & 0,0 & 154 \\
Gama & 71 & 27,8 & & 3 & 100,0 & 74 \\
Planaltina & 30 & 11,8 & & 0 & 0,0 & 30 \\
\hline Total & $\mathbf{2 5 5}$ & $\mathbf{1 0 0 , 0}$ & $\mathbf{3}$ & $\mathbf{1 0 0 , 0}$ & $\mathbf{2 5 8}$ \\
\hline \% & $\mathbf{9 8 , 8 4}$ & - & $\mathbf{1 , 1 6}$ & - & $\mathbf{1 0 0}$ \\
\hline
\end{tabular}

TABELA 3

Distribuição das espécies de flebotomíneos, vetores da leishmaniose tegumentar, por local de coleta. Distrito Federal, no período de maio de 2006 a junho de 2007.

\begin{tabular}{|c|c|c|c|c|c|c|c|c|}
\hline \multirow{2}{*}{$\begin{array}{l}\text { Locais de coleta } \\
\text { Espécies }\end{array}$} & \multicolumn{2}{|c|}{ Intradomicílio } & \multicolumn{2}{|c|}{ Peridomicílio } & \multicolumn{2}{|c|}{ Vegetação } & \multicolumn{2}{|c|}{ Total } \\
\hline & $\mathrm{n}^{0}$ & $\%$ & $\mathrm{n}^{0}$ & $\%$ & $\mathrm{n}^{\mathrm{o}}$ & $\%$ & $\mathrm{n}^{\underline{0}}$ & $\%$ \\
\hline Lutzomyia whitmani & 10 & 4,1 & 145 & 59,2 & 90 & 36,7 & 245 & 100,0 \\
\hline Lutzomyia flaviscutellata & 0 & 0 & 0 & 0 & 3 & 100 & 03 & 100,0 \\
\hline
\end{tabular}

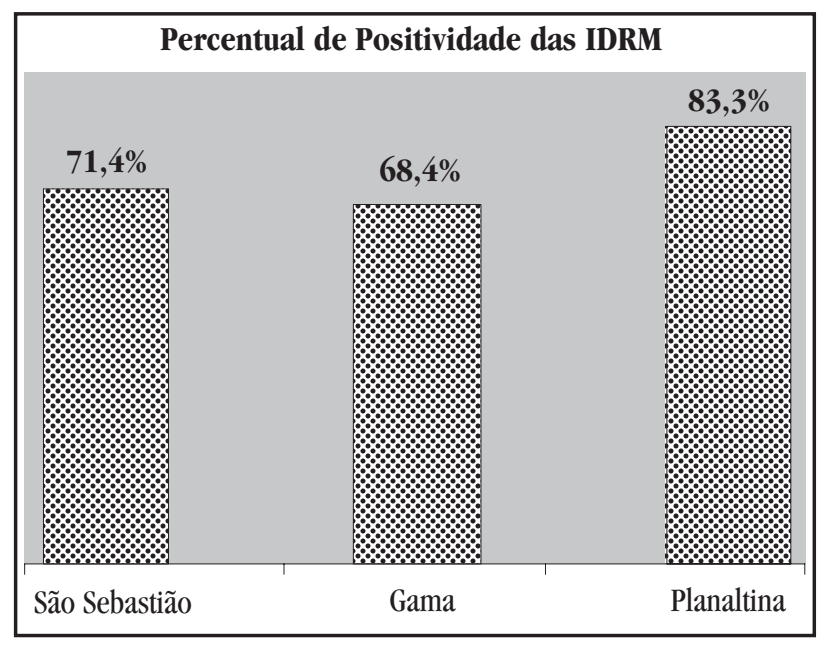

FIGURA 2

Percentuais de positividade encontrados nas intradermorreações de Montenegro de moradores da mesma casa e vizinhos de quatro pacientes atendidos no Hospital Universitário de Brasília, de acordo com a região administrativa estudada, no período de agosto de 2006 a junho de 2007.

\section{DISCUSSÃo}

Observou-se rigorosamente o pré-requisito de incluir apenas os pacientes com LTA que informaram não terem saído dos limites do DF por um tempo mínimo de seis meses, anteriores ao aparecimento da lesão de pele. Admitindo que o período de incubação da doença varie de 18 dias a 4 meses $^{111}$, acredita-se, portanto, que se trate de casos autóctones.

A maioria dos pacientes portadores de LTA atendidos no DF procede de outros Estados, predominando Goiás, Minas Gerais, Bahia e estados do Nordeste e Norte ${ }^{1516}$.

ODistrito Federal sofreu crescimento populacional descontrolado e exagerado. Segundo dados de 1996, a população que era de 1.817.000 habitantes, em 2006 totalizava 2.383.784 (IBGE). Isto representa um crescimento de 30,2\% em 10 anos. Grande parte desta população veio de outros estados, sobretudo da Região Nordeste, e se instalou em condições inadequadas de habitação e saneamento, causando grandes alterações sociais. Fazendo parte desta população, podem ter migrado pacientes com leishmaniose que aqui se instalaram, inclusive com seus animais de estimação, numa convivência com potenciais transmissores da doença, facilitando, desta forma, a infecção peridomiciliar. Além disso, deve ser considerado que o DF está localizado dentro do estado de Goiás e faz fronteira com Minas Gerais, estados com alta incidência de $\operatorname{LTA}^{910} 16$.

Houve predominância da incidência de LTA em homens, faixa etária entre 11 e 58 anos, dados semelhantes aos estudos realizados em outros estados ${ }^{2021} \mathrm{e}$, em período anterior, no $\mathrm{DF}^{14}$. Foram acometidas duas crianças até 12 anos de idade. Levanta-se a hipótese de transmissão peri/intradomicíliar reforçada pelos achados de flebotomíneos no peri/intradomicílio e 21,7\% de positividade de IFI em crianças até 10 anos.

Todos pacientes do DF apresentaram lesão cutânea única com predomínio de lesão ulcerada, semelhante a dados obtidos em estudos anteriores, nos quais predominavam pacientes de outros estados ${ }^{14}$. No que se refere à localização das lesões, houve predomínio destas em membros superiores (6 de 10 doentes) quando, normalmente, são os membros inferiores mais atingidos. Talvez isto possa ser justificado por hábitos no modo de vestir dos pacientes ou peculiaridades da altura do vôo dos transmissores ${ }^{214}$. Quanto à RA de procedência dos pacientes, em 1999, houve maior incidência na RA Planaltina ${ }^{15}$. Em 2003, houve relato de surto na RA São Sebastião ${ }^{12}$. Neste estudo, a maioria procedia da RA Planaltina (DF).

Dos exames utilizados para confirmação de diagnóstico, a IDRM teve o maior percentual de positividade, como já esperado. A positividade das culturas e pesquisa de amastigotas foi relativamente elevada ( $80 \%$ e $70 \%$, respectivamente). Isto pode ter acontecido porque a maioria $(80 \%)$ dos casos tinha até 6 meses de evolução de doença ${ }^{2}{ }^{14}{ }^{18}$.

A positividade das culturas, na maioria dos casos, foi coincidente com a positividade da pesquisa de amastigotas em esfregaço (70\% x 60\%), havendo concordância entre os exames parasitológicos.

Nos testes realizados entre familiares e vizinhos dos pacientes, o elevado $(71,9 \%)$ percentual de positividade das IDRM sugere infecção subclínica por leishmania, acreditandose na possibilidade de transmissão local. As IFI foram $100 \%$ negativas em exames repetidos duas vezes. Em estudo anterior, foi encontrado percentual de $33,3 \%$ de positividade ${ }^{19}$.

0 maior número de familiares e vizinhos de pacientes foi encontrado na RA Gama, seguido da RA São Sebastião e Planaltina. A região administrativa Planaltina contribuiu com o maior número de pacientes com ITA e o maior percentual de positividade das IDRM realizadas em moradores. 0 percentual de positividade das IDRM da RA Planaltina foi seguido pela RA São Sebastião e pela RA Gama, respectivamente. 0 menor percentual da RA Gama pode ser pelo predomínio de crianças, menos expostas às atividades de risco para contrair a doença. 
Cinco culturas isoladas foram identificadas, por PCR e anticorpos monoclonais, como Leishmania (Viannia) braziliensis. Isto confirma resultados anteriores com predominância deste parasito ${ }^{17}$, corroborado por aspectos clínicos, resultados laboratoriais e do tratamento dos pacientes e os achados da fauna flebotomínica.

Houve boa resposta do tratamento ao NMG endovenoso, como esperado na doença recente causada pela Leishmania (Viannia) braziliensis, e também, de maneira mais surpreendente, boa resposta de dois casos ao NMG intralesional.

Lutzomyia whitmani, representou 98,9\% das espécies vetoras capturadas e foi encontrada nas três regiões administrativas estudadas. Sua presença foi maior $(59,2 \%)$ no peridomicílio. 0 achado no intra/peridomicílio é um indicativo de que está ocorrendo transmissão de LTA no DF, também, em ambiente domiciliar. Lutzomyia flaviscutellata foi identificada apenas na região administrativa Gama em ambiente de cerrado tipo vereda com 1,2 \% do total dos flebotomíneos vetores (Tabela 2).

Estas duas espécies de flebotomíneos encontradas correspondem a registros feitos anteriormente de espécies encontradas no $\mathrm{DF}^{14}$, constituindo preocupação, hoje, a presença de flebotomíneos no peri/intradomicílio ${ }^{4}$.

Houve boa interação entre a equipe da UnB e a da SES, DF. Isto facilita o envio dos dados dos pacientes à Secretaria, permitindo ações mais precoces de controle nas áreas de maior risco de transmissão da doença e prevenindo futuras infecções.

Conclui-sequedevehavertransmissão daLTAnoDF,provavelmente, envolvendo o peridomicílio e as espécies predominantes de flebotomíneo e leishmania são Lutzomia whitmani e Leishmania (Viannia) braziliensis, respectivamente.

Além de aprofundar no estudo de todos os fatores que envolvem a transmissão de ITA no DF é necessária a conscientização da população sobre a transmissão, de procurar tratamento precoce para feridas que não se cicatrizam com a participação ativa da população para o efetivo controle da doença.

\section{AGRADECIMENTOS}

Ao Tércio Rodrigues Pereira pelo apoio ao trabalho de campo e realização dos exames.

\section{REFERÊNCIAS}

1. Antonio LF, Fagundes A, Schubach AO, Freitas L, Marzochi KBF. Padronização clínica do teste de Montenegro. Revista da Sociedade Brasileira de Medicina Tropical 40(supl I):138, 2007.

2. Carvalho MLR, Fontes CJF, Hueb M, Guedes AM, Afonso LCC, Melo MN. Leishmaniose Tegumentar no estado de Mato Grosso (Brasil): estudo clínico, laboratorial e terapêutico. Anais Brasileiros de Dermatologia 1:45-56, 2002.

3. Ferreira MP, Roselino AMF, Nascimento MMP, Aires JM, Figueiredo JFC. Sensitivity of an immunoenzymatic test for the detection of Anti-L. braziliensis antibodies compared to other tests used for the diagnosis of american cutaneous leishmaniasis. Revista do Instituto de Medicina Tropical de São Paulo 4: 215-217, 2006.

4. Gomes AC, Rabello EX, Santos JLF, Galati EAB. Aspectos ecológicos da leishmaniose tegumentar americana. 2 Ecótopo artificial como abrigo de Psychodopygus intermedius e observações sobre alimentação e reprodução sob influência de fatores físicos naturais. Revista de Saúde Pública 16: 149-159, 1982.

5. Gontijo B, Carvalho MLR. Leishmaniose tegumentar americana. Revista da Sociedade Brasileira de Medicina Tropical 1:71-80, 2003.

6. Grimaldi Junior G, McMahon-Pratt D. Monoclonal antibodies for the identification of new world Leishmania species. Memórias do Instituto 0swaldo Cruz 91:37-42, 1996.

7. Marzochi MCA, Coutinho SG, Sabroza PC, de Souza WJ. Indirect immunofluorescence reaction and intradermoreaction for American cutaneous leishmaniasis in residents of the Jacarepaguá region (Rio de Janeiro): comparative study of results observed in 1974 and 1978. Revista do Instituto de Medicina Tropical 22:149-155, 1980.

8. Menezes JAS, Moura NFO, Elkhoury ANSM, Alves WA, Souza M, Oliveira SM, Dantas MCS. 0 diagnóstico da leishmaniose tegumentar americana no Brasil, a partir de análise do Sistema Nacional de Informação de Agravos. Revista da Sociedade Brasileira de Medicina Tropical 49 (supl 1): 124, 2007.

9. Ministério da Saúde. Leishmaniose tegumentar americana: distribuição de casos confirmados, por Unidade Federada. Brasil, 1980-2005. Disponível em: http://portal.saude.gov.br/portal/arquivos/pdf/leishmaniose_2006. pdf

10. Ministério da Saúde. Manual de vigilância da leishmaniose tegumentar americana. Brasília, DF, 2007.

11. Pessoa SB, Barreto MP. Leishmaniose tegumentar americana. Ministério da Educação e Saúde, Rio de Janeiro, 1948.

12. Porto C. Autoctonia da leishmaniose tegumentar americana no Distrito Federal. Estudo em seres humanos e cães na cidade de São Sebastião. Dissertação de Mestrado, Universidade de Brasília, Brasília, 2004.

13. Sampaio RNR. Investigação epidemiológica da autoctonia da leishmaniose tegumentar americana no Distrito Federal. Anais Brasileiros de Dermatologia 74: 197, 1999 .

14. Sampaio RNR, Paula CDR. Leishmaniose Tegumentar Americana no Distrito Federal. Revista da Sociedade Brasileira de Medicina Tropical 5: 523-528, 1999.

15. Sampaio RNR, Rocha RAA, Marsden PD, Cuba CC, Barreto AC. Leishmaniose tegumentar americana. Casuística do Hospital Escola da UnB. Anais Brasileiros de Dermatologia 55: 69-76, 1980.

16. Sampaio RNR, Sampaio JHD, Tauil PL, Nogueira LSC, Borges KT, Name RQ. Estudo clínico, epidemiológico e terapêutico de 402 pacientes, com leishmaniose tegumentar americana, atendidos no Hospital Universitário de Brasília, DF, Brasil. Anais Brasileiros de Dermatologia 3:249-254, 2005.

17. Santos GM, Sampaio RNR. A Leishmania Viannia braziliensis é a principal espécie causadora da Leishmaniose tegumentar no DF. Revista da Sociedade Brasileira de Medicina Tropical 40 (supl I): 112, 2007.

18. Sessa PA, Falqueto A, Barros GC, Varejão JBM. Resultados da reação de Montenegro em pacientes com leishmaniose tegumentar americana, autóctones do Estado do Espírito Santo. Revista da Associação Médica Brasileira 37: 115-118, 1991.

19. Silva TAV, John FP, Sampaio, RNR. Autoctonia da leishmaniose tegumentar americana no Distrito Federal-Brasil. The Brazilian Journal of Infectious Diseases 7 (supl I): 153, 2003.

20. Silveira FT, Lainson R, Corbett CEP. Clinical and immunopathological spectrum of american cutaneous leishmaniasis with special reference to the disease in amazonian Brazil - a review. Memórias do Instituto Oswaldo Cruz 3: 239-251, 2004 .

21. Vieira ML, Jacobina RR, Soares NM. Casos de leishmaniose em pacientes atendidos nos centros de saúde e hospitais de Jacobina-BA no período de 2001 a 2004. Revista Baiana de Saúde Pública 1: 102-114, 2007. 